\title{
Direct Numerical Simulation DNS: Maximum Error as a Function of Mode Number
}

\author{
L. Jameson
}

June 1, 2000

U.S. Department of Energy

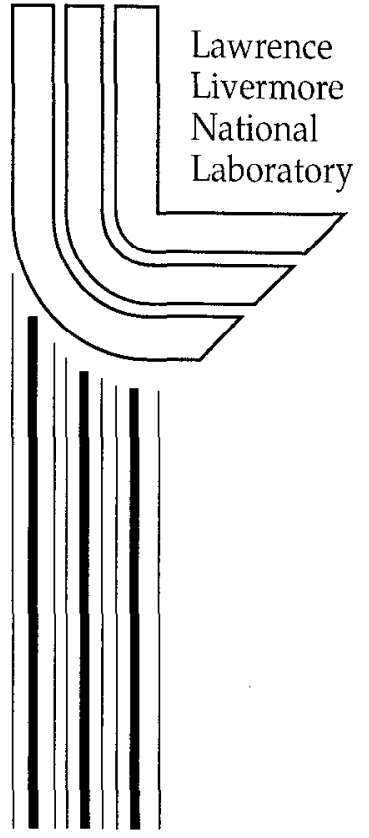




\section{DISCLAIMER}

This document was prepared as an account of work sponsored by an agency of the Uniled States Government. Neither the United States Government nor the University of California nor any of their employees, makes any warranty, express or implied, or assumes any legal liability or responsibility for the accuracy, completeness, or usefulness of any information, apparatus, product, or process disclosed, or represents that its use would not infringe privately owned rights. Reference herein to any specific commercial product, process, or service by trade name, trademark, manufacturer, or otherwise, does not nccessarily constitute or imply its endorsement, recommendation, or favoring by the United States Government or the University of California. The views and opinions of authors expressed herein do not necessarily state or reflect those of the United States Government or the University of California, and shall not be used for advertising or product endorsement purposes.

Work performed under the auspices of the U.S. Department of Energy by the University of California Lawrence Livermore National Laboratory under Contract W-7405-Eng-48.

This report has been reproduced directly from the best available copy.

Available to DOE and DOE contractors from the Office of Scientific and Technical Information P.O. Box 62, Oak Ridge, TN 37831

Prices available from (423) 576-8401 http://apollo.osti.gov/bridge/

Available to the public from the National Technical Information Service

U.S. Department of Commerce 5285 Port Royal Rd., Springfield, VA 22161 http://www.ntis.gov/

$\mathrm{OR}$

Lawrence Livermore National Laboratory Technical Information Department's Digital Library http://www.Ilnl.gov/tid/Library.html 


\title{
Direct Numcrical Simulation DNS: Maximum Error as a Function of Mode Number
}

\author{
Leland Jamcson
}

\begin{abstract}
Numerical errors can be characterized in terms of algebraic polynomial approximation of a sine wave. The magnitude of the error will, therefore, depend on the energy at each mode in a Fourier expansion. Flows with a great deal of energy in the highest modes, such as Turbulencc, are therefore the most difficult to approximate.
\end{abstract}




\section{Contents}

1 Introduction 1

2 Spectrum and Errors 5

2.1 Finding the Maximum Spatial Error . . . . . . . . . . 6

2.1.1 Maximum Spatial Error for $c_{k}=e^{-d k}$. . . . . . . . 7

2.1.2 Maximum Spatial Error for $c_{k}=e^{-d k^{2}}$. . . . . . . . . 7

2.1.3 Algebraic Decaying Spectrum: $c_{k}=k^{-m}$. . . . . . 8

2.1.4 Maximum Spatial Error for Turbulent Flow: $c_{k}=k^{-5 / 3} e^{-c k / k_{k o l}} . \quad 8$

< 2.1.5 Maximum Spatial Error for $c_{k}=k^{m} e^{-a k^{b}} \ldots \ldots 8$

2.2 Finding the Maximum Temporal Error . . . . . . . . . . 8

2.2.1 Maximum Temporal Error for $c_{k}=e^{-d k} \ldots \ldots . . .99$

$\begin{array}{llr}3 \text { Conclusion } & 16\end{array}$

$\begin{array}{lll}4 & \text { Bibliography } & 17\end{array}$

\section{List of Figures}

1 Differentiation error for various schemes applied to data with a spectrum $c_{k}=k^{-5 / 3} e^{-5 k / k_{u}}$. FD2 (labeled with o), FD4 (labeled with triangle), FD8 (labeled with a diamond) and a Fourier spectral method (labeled with an asterisk). Actual pointwise errors and theoretical upper bound curves. The number of grid points is 32 hence one can observe aliasing in the spectral curve. . . . . . . . . . . . . . . 10

2 Differentiation error for various schemes applied to data with spectrum $c_{k}=e^{-k}$. FD2 (labeled with o), FD4 (labeled with triangle), FD8 (labeled with a diamond) and a Fourier spectral method (labeled with an asterisk). Actual pointwise errors and theoretical upper bounds. Theoretical maximum is given by the plus line. The number of grid points is 64 , therefore no aliasing can be seen in the spectral curve. . . . . . . . . . . 11 
3 Differentiation error for various schemes applied to data with spectrum $c_{k}=e^{-k^{2} / 10}$. FD2 (labeled with o), FD4 (labeled with triangle), FD8 (labeled with a diamond) and a Fourier spectral method (labeled with an asterisk). Actual pointwise errors and theoretical upper bounds. Theoretical maximum is given by the plus line. The number of grid points is 64 , therefore no aliasing is seen.

4 Here we observe the errors for Runge-Kutta time advancement. Errors are shown for RK2, RK4, and RK6. $c_{k}=e^{-k}$. 13

5 Error for FD2, FD4, FD8, and spectral. $c_{k}=1 / k$. Note that this spectrum would be similar to that of a shock. Note how the error is always increasing. . . . . . . . . . . 14

6 Error for RK2, RK4, and RK6. $c_{k}=1 / k$ Again, the spectrum is that of a shock. 


\section{Introduction}

Direct Numerical Simulation, DNS, is generally thought of as resolving all scales in a calculation. Scales are usually correlated in a global sense to the highest mode in a Fourier series expansion and one generally considers spectral methods as the best numerical candidate to obtain true DNS. In other words, at a given time in calculation the fields will have a Fourier Scries cxpansion of the form,

$$
f(x)=\sum_{k=0}^{N / 2} c_{k} e^{i k x},
$$

and one often says that the calculation is "resolved" if, in the case of spectral methods, there are $N$ grid points and, hence, $N / 2$ modes. The critical point to be made here is that the temporal discretization is almost always ignored in this context. But, the temporal discretization is as important as the spatial discretization and in order to state that a given calculation is "resolved" it must be resolved in both space and time and not simply space, as is often miss-stated. For spectral calculations a common temporal discretization is through the class of Runge-Kutta schemes. Runge-Kutta schemes are very reliable and probably the best choice for spectral discretization, but they are fundamentally based on an algebraic polynomial approximation through a Taylor series. In the early 80's, [?], there was some activity in using spectral methods for temporal advancement as well as spatial discretization, but such methods never became widely used. So, even with spectral methods we are confronted with the fact that the faithfulness of our DNS will be limited to the quality of our algebraic polynomial approximation in time to our Fourier expansion. 


\section{Spectrum and Errors}

Our primary concern here is the numerical error committed at a given step during a simulation. During a given step can assume that the spectrum is constant with respect to time. Of course, over an entire simulation, this will certainly not be the case, but for one individual time step it is a fair assumption.

A function of interest will have a certain spectrum which most likely decays with increasing mode. That is, we can expect our function to have the form,

$$
f(x)=\sum_{k \leq N / 2} c_{k} e^{i k x}
$$

where we can expect that the spectrum represented by $c_{k}$ will decay. For example, it might be $c_{k}=e^{-k}$ or perhaps $c_{k}=e^{-k^{2}}$. We can see from ([2]) that the truncation error was calculated for each mode and that the truncation error grows with mode. However, when we add in the spectrum $c_{k}$ then we can expect that the error will peak at some wavenumber between the lowest wavenumber and the highest. To illustrate further, suppose our function is,

$$
f(x)=\sum_{k \leq N / 2} e^{-\left(k^{2}\right)} e^{i k x}
$$

from which we get that,

$$
f^{(p)}(x) \sum_{k \leq N / 2}(i k)^{p} e^{-\left(k^{2}\right)} e^{i k x}
$$

and we can see that this expression is bounded above by

$$
B(k)=k^{p} e^{-\left(k^{2}\right)},
$$

which is the product of the spectrum and $k^{p}$ which comes from the differentiation. This function, $B(k)$, will have a maximum value at a given wavenumber. It is this maximum error that we focus on obtaining in this manuscript. Obtaining the maximum value for $B(k)$ for various flows is critical to obtaining estimates on the reliability of a given calculation. 


\subsection{Finding the Maximum Spatial Error}

Here we will focus on the case of finding these maximum error values for finite difference operators. Other types of differentiation operators such as compact, finite elements, etc. are a straightforward extension of the approach outlined here.

Now consider the expression derived in ([2]) for the upper bound on the differentiation error,

$$
E(h, p, k)=h^{p}((p / 2) !)^{2} \frac{k^{p+1}}{(p+1) !},
$$

where $h$ denotes the distance between grid points (assumed uniform here), $p$ denotes the numerical order of accuracy, and $k$ denotes the wavenumber. Recall this expression was derived for $c_{k}=1$ for all $\mathrm{k}$. For an arbitrary spectrum $c_{k}$ the upper bound on the error becomes,

$$
E(h, p, k)=h^{p}((p / 2) !)^{2} \frac{k^{p+1}}{(p+1) !} c_{k} .
$$

Now let $h$ and $p$ be fixed and find the maximum value of this error with respect to the mode number $k$. In this case we get,

$$
E(k)=\operatorname{Const}(h, p) k^{p+1} c_{k},
$$

where,

$$
\operatorname{Const}(h, p)=\frac{h^{p}((p / 2) !)^{2}}{(p+1) !} .
$$

Consider $k$ as a continuous variable and differentiate the above expression for $E(k)$ with respect to $k$ to get,

$$
E^{\prime}(k)=\operatorname{Const}(h, p)\left((p+1) k^{p} c_{k}+k^{p+1} c_{k}^{\prime}\right) .
$$

Setting this expression equal to zero, one gets,

$$
(p+1) c_{k}+k c_{k}^{\prime}=0
$$

which is the key expression that must be solved in order to find the maximum value of the error. Note that this error does depend not only on the spectrum 
and the wavenumber but also on the numerical order of the scheme at hand. If perchance the spectrum is time evolving, i.e., $c_{k}=c_{k}(t)$ then the above expression would certainly hold for each value of time to get,

$$
(p+1) c_{k}(t)+k \frac{d}{d k} c_{k}(t)=0
$$

In the next few subsections we will find the maximum errors for various spectra of interest.

\subsubsection{Maximum Spatial Error for $c_{k}=e^{-d k}$.}

For $c_{k}=e^{-d k}$ we see that,

$$
((p+1)-d k) e^{-d k}=0
$$

or simply,

$$
k=\frac{p+1}{d}
$$

And, we expect that our maximum error will be bounded above by,

$$
\operatorname{Emax}(h, p, d)=h^{p}\left(\left(\frac{p}{2}\right) !\right)^{2} \frac{((p+1) / d)^{p+1}}{(p+1) !} e^{-(p+1)}
$$

\subsubsection{Maximum Spatial Error for $c_{k}=e^{-d k^{2}}$.}

For our second spectrum $c_{k}=e^{-d k^{2}}$ we arrive al,

$$
\left((p+1)-2 d k^{2}\right) e^{-d k^{2}}=0
$$

or the maximum error will occur at

$$
k=\sqrt{\frac{p+1}{2 d}}
$$

In the same manner as above, one can find the maximum error to be,

$$
\operatorname{Emax}(h, p, d)=h^{p}\left(\left(\frac{p}{2}\right) !\right)^{2} \frac{\left(\frac{p+1}{2 d}\right)^{(p+1) / 2}}{(p+1) !} e^{-(p+1) / 2}
$$




\subsubsection{Algebraic Decaying Spectrum: $c_{k}-k^{-m}$.}

Finally, one of our most important examples is the case when $c_{k}=k^{-m}$. From above we see that the character of the error, $E(k)=\operatorname{Const}(h, p) k^{p+1} c_{k}$, changes drastically depending on if $m$ is less than or greater than $p+1$. For $m \leq p+1$ we see that the error bound will always grow with respect to $k$ which means that our errors will be dominated by the error at the largest wavenumber. On the other hand, if $m \geq p+1$ then the error is a monotonically decreasing function with respect to $k$ and our errors are dominated by the errors at the lowest mode. Neither situation is desirable since the errors at these extremes are quite large.

\subsubsection{Maximum Spatial Error for Turbulent Flow: $c_{k}=k^{-5 / 3} c^{-c k / k_{k o l}}$.}

One possibility for a turbulent spectrum is, $c_{k}=k^{-5 / 3} e^{-c k / k_{k o l}}$, ([3]). The calculation for this spectrum yields,

$$
k=\frac{k_{k o l}}{c}(5 / 3-(p+1)) .
$$

\subsubsection{Maximum Spatial Error for $c_{k}=k^{m} e^{-a k^{b}}$}

With this spectrum we first find the derivative of $c_{k}$ to get,

$$
c_{k}^{\prime}=e^{a k^{b}} k^{m}{ }^{1}\left(m-a b k^{b}\right) .
$$

Using this expression we get,

$$
k^{m} e^{-a k^{b}}\left(p+m-a b k^{b}+1\right)=0,
$$

or the maximum error occurs at mode,

$$
k=\left(\frac{p+m+1}{a b}\right)^{1 / b} .
$$

\subsection{Finding the Maximum Temporal Error}

Deriving a similar expression for the temporal error, one finds that the maximum temporal error for Runge-Kutta Schemes occurs at the mode $k$ satisfying,

$$
p c_{k}+k c_{k}^{\prime}=0 .
$$


In a manner completely analogous to that above, one can find the maximum temporal error for the Runge-Kutta class of time advancement for various spectra. We find this error for one representative spectrum.

\subsubsection{Maximum Temporal Error for $c_{k}=e^{-d k}$.}

We can easily find that the maximum error occurs at,

$$
k=\frac{p}{d},
$$

and one finds the maximum error to be,

$$
\text { Emaxtime }=\frac{(\Delta t)^{p-1}}{p !}\left(\frac{p}{d}\right)^{p} e^{-p}
$$




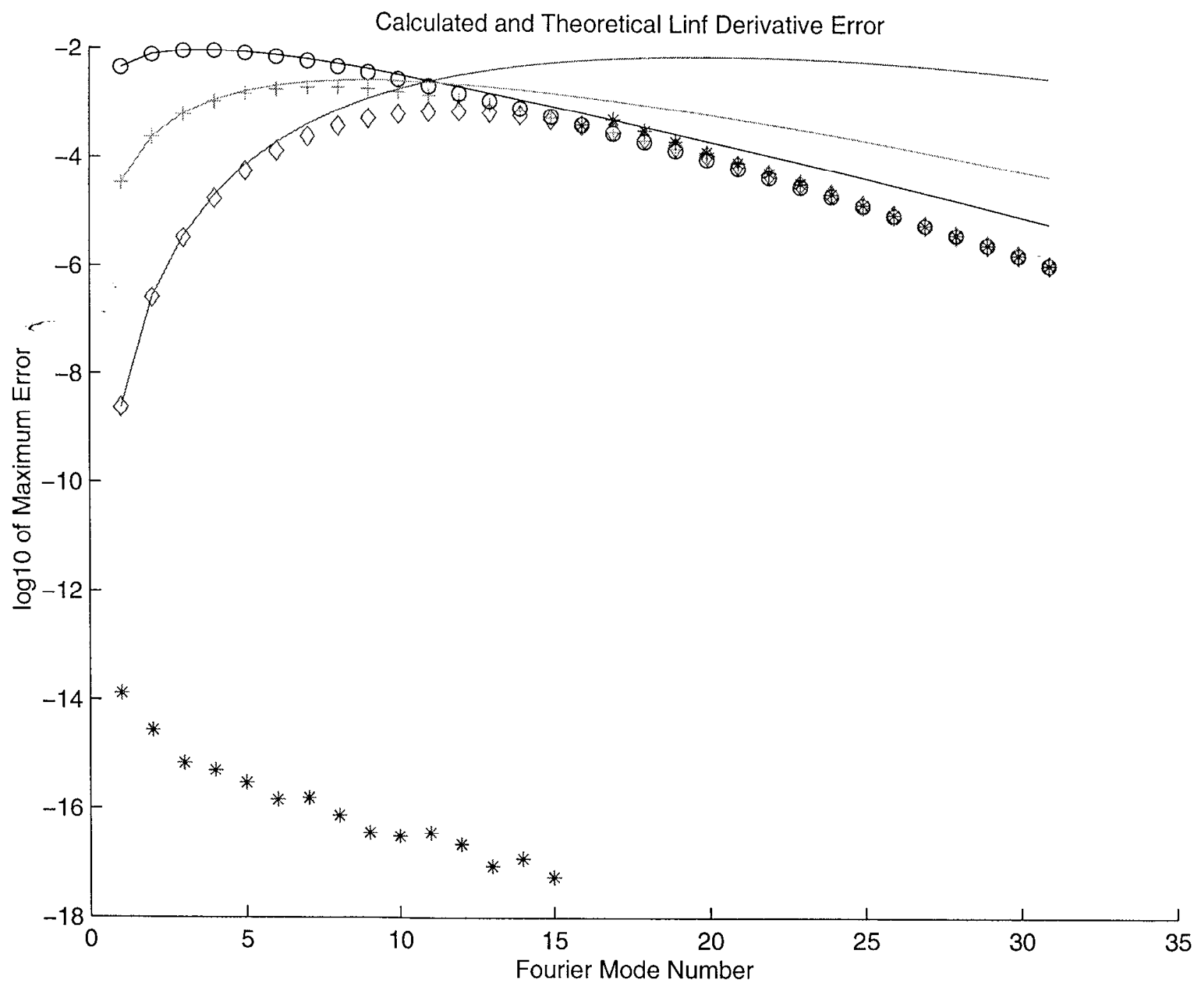

Figure 1: Differentiation error for various schemes applied to data with a spectrum $c_{k}-k^{-5 / 3} e^{-5 k / k_{0}}$. FD2 (labeled with o), FD4 (labcled with triangle), FD8 (labeled with a diamond) and a Fourier spectral method (labeled with an asterisk). Actual pointwise errors and theoretical upper bound curves. The number of grid points is 32 hence one can observe aliasing in the spectral curve. 


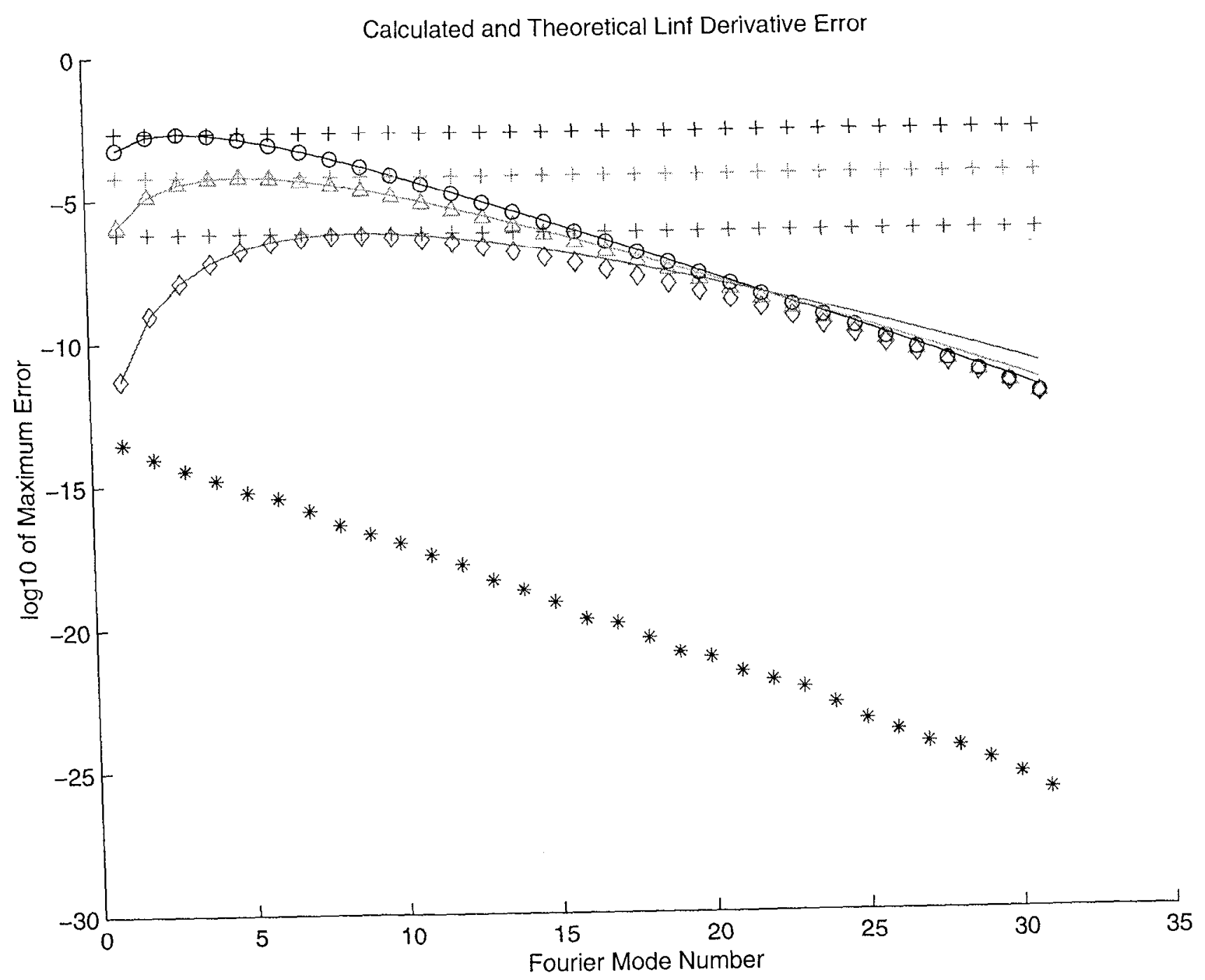

Figure 2: Differentiation error for various schemes applied to data with spectrum $c_{k}=e^{-k}$. FD2 (labeled with o), FD4 (labeled with triangle), FD8 (labeled with a diamond) and a Fourier spectral method (labeled with an asterisk). Actual pointwise errors and theoretical upper bounds. Theoretical maximum is given by the plus line. The number of grid points is 64 , therefore no aliasing can be seen in the spectral curve. 


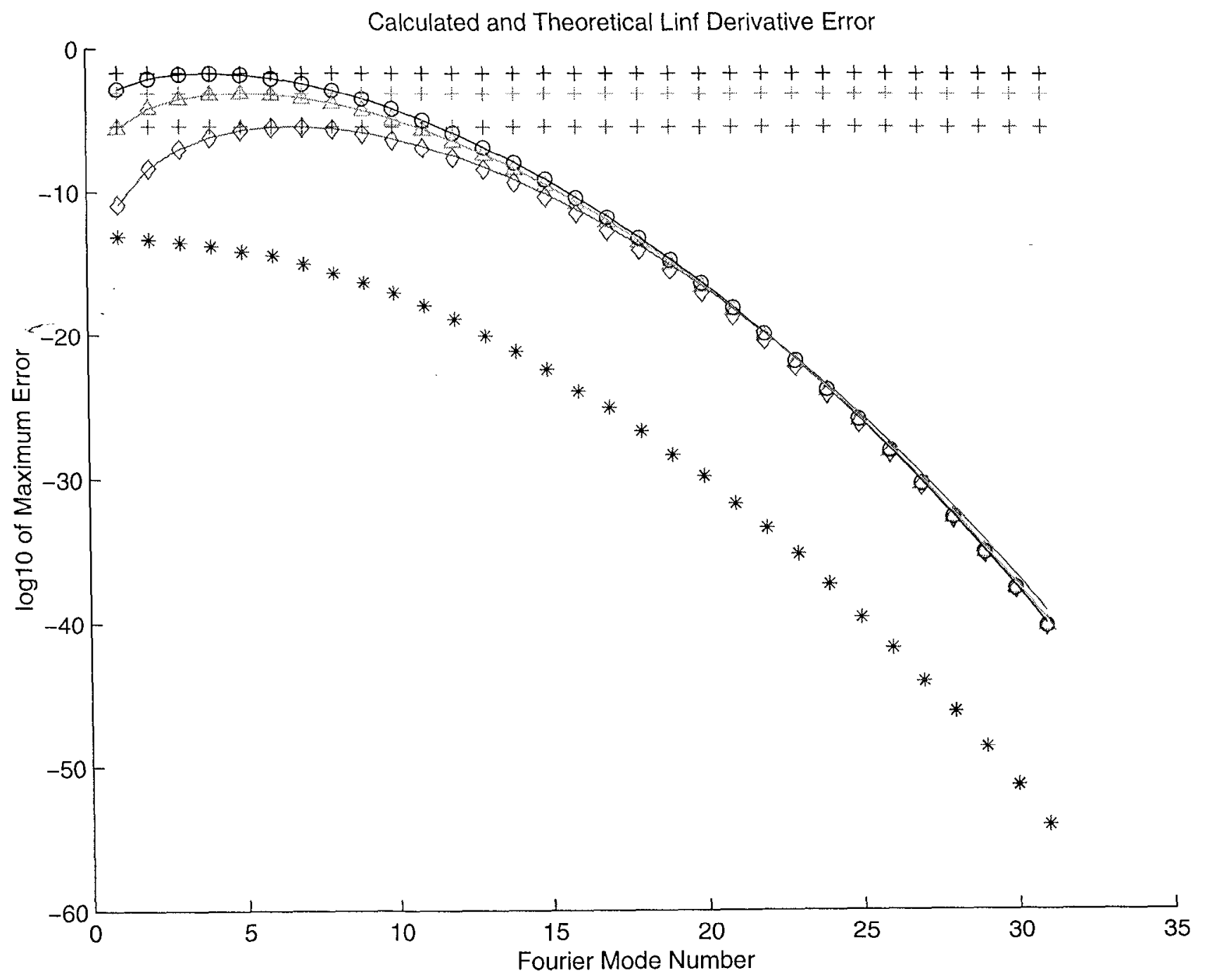

Figure 3: Differentiation error for various schemes applied to data with spectrum $c_{k}=e^{-k^{2} / 10}$. FD2 (labeled with o), FD4 (labeled with triangle), FD8 (labeled with a diamond) and a Fourier spectral method (labeled with an asterisk). Actual pointwise errors and theoretical upper bounds. Theoretical maximum is given by the plus line. The number of grid points is 64 , therefore no aliasing is seen. 


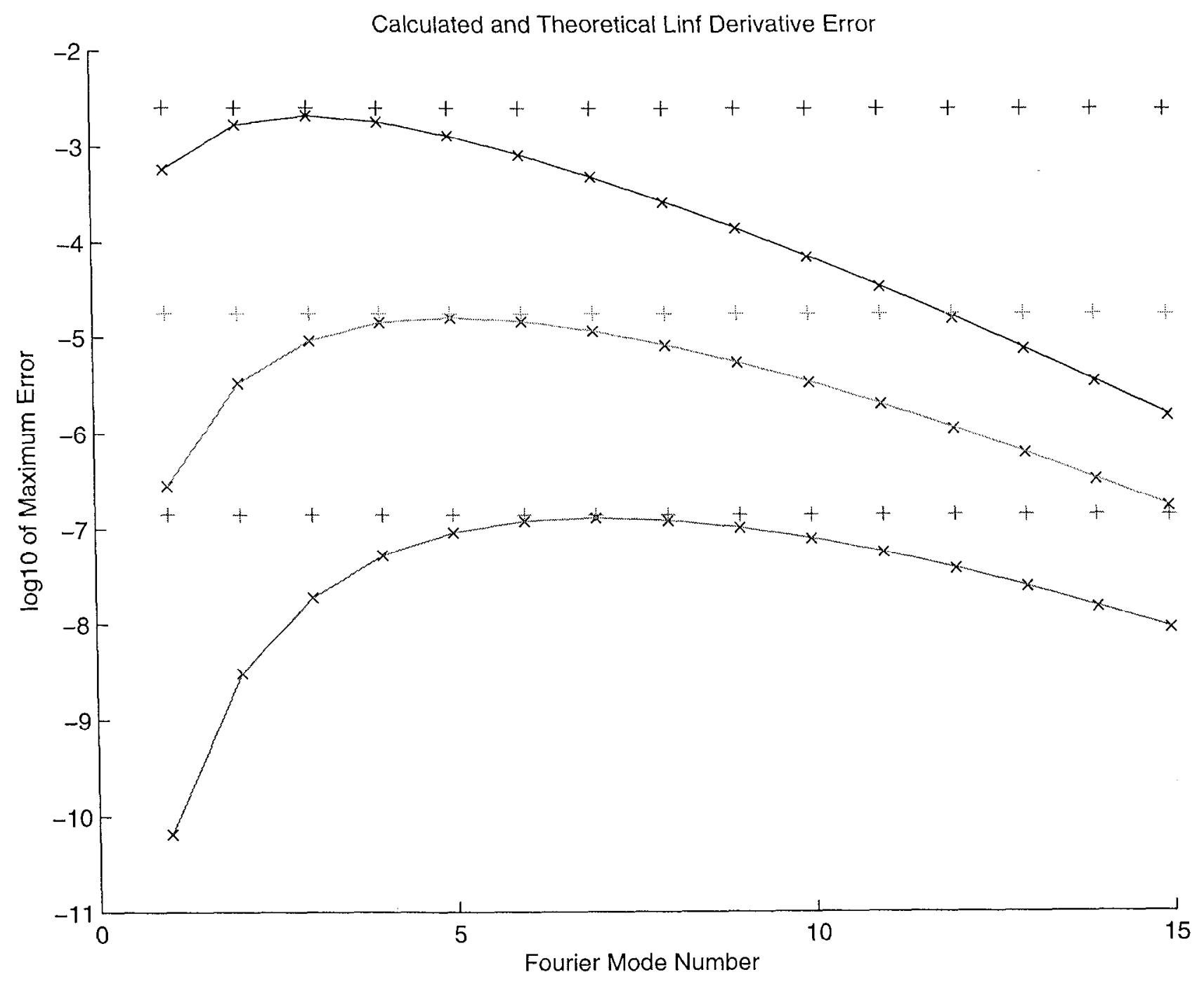

Figure 4: Here we observe the errors for Runge-Kutta time advancement. Errors are shown for RK2, RK4, and RK6. $c_{k}=e^{-k}$ 


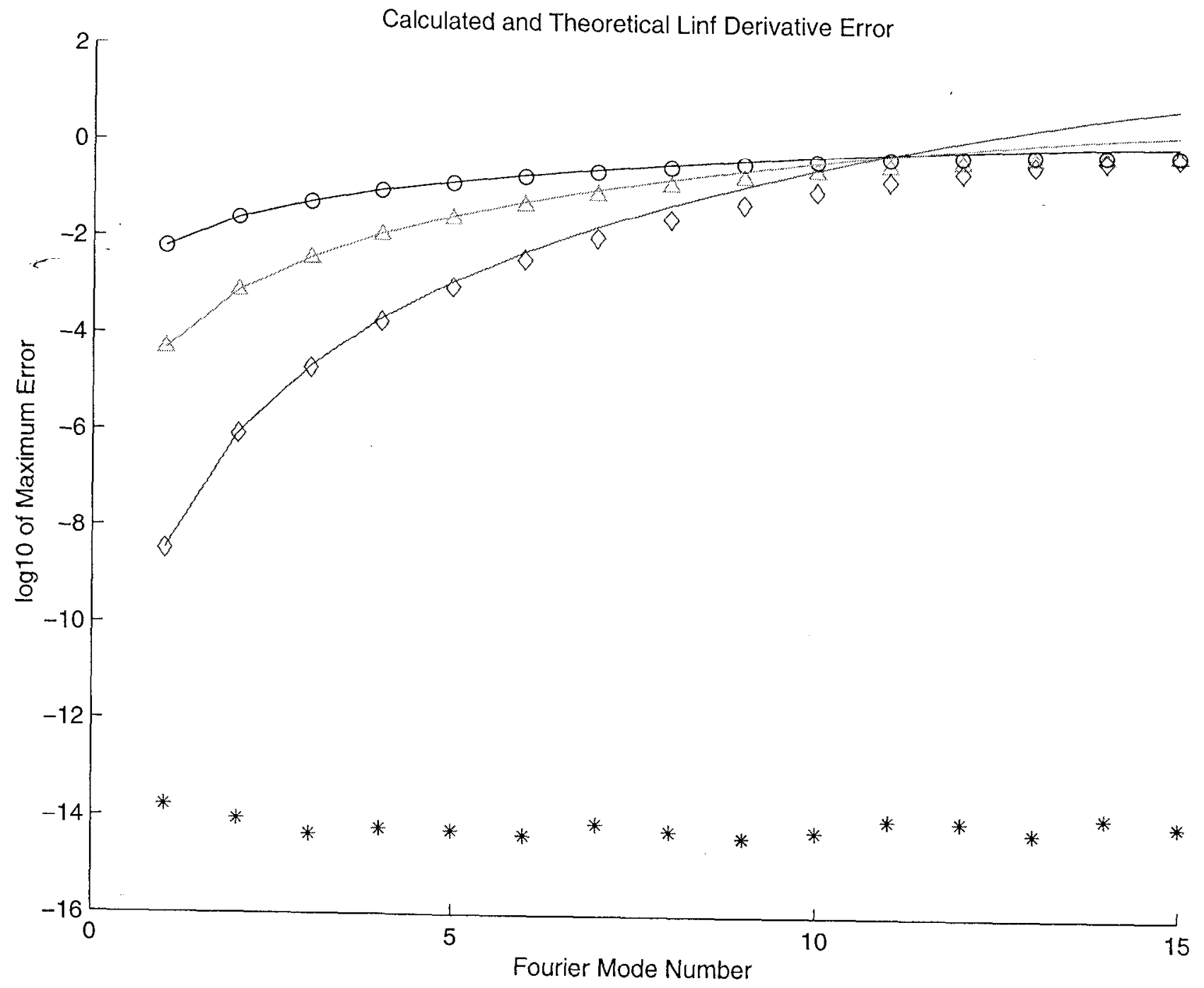

Figure 5: Error for FD2, FD4, FD8, and spectral. $c_{k}=1 / k$. Note that this spectrum would be similar to that of a shock. Note how the error is always increasing. 


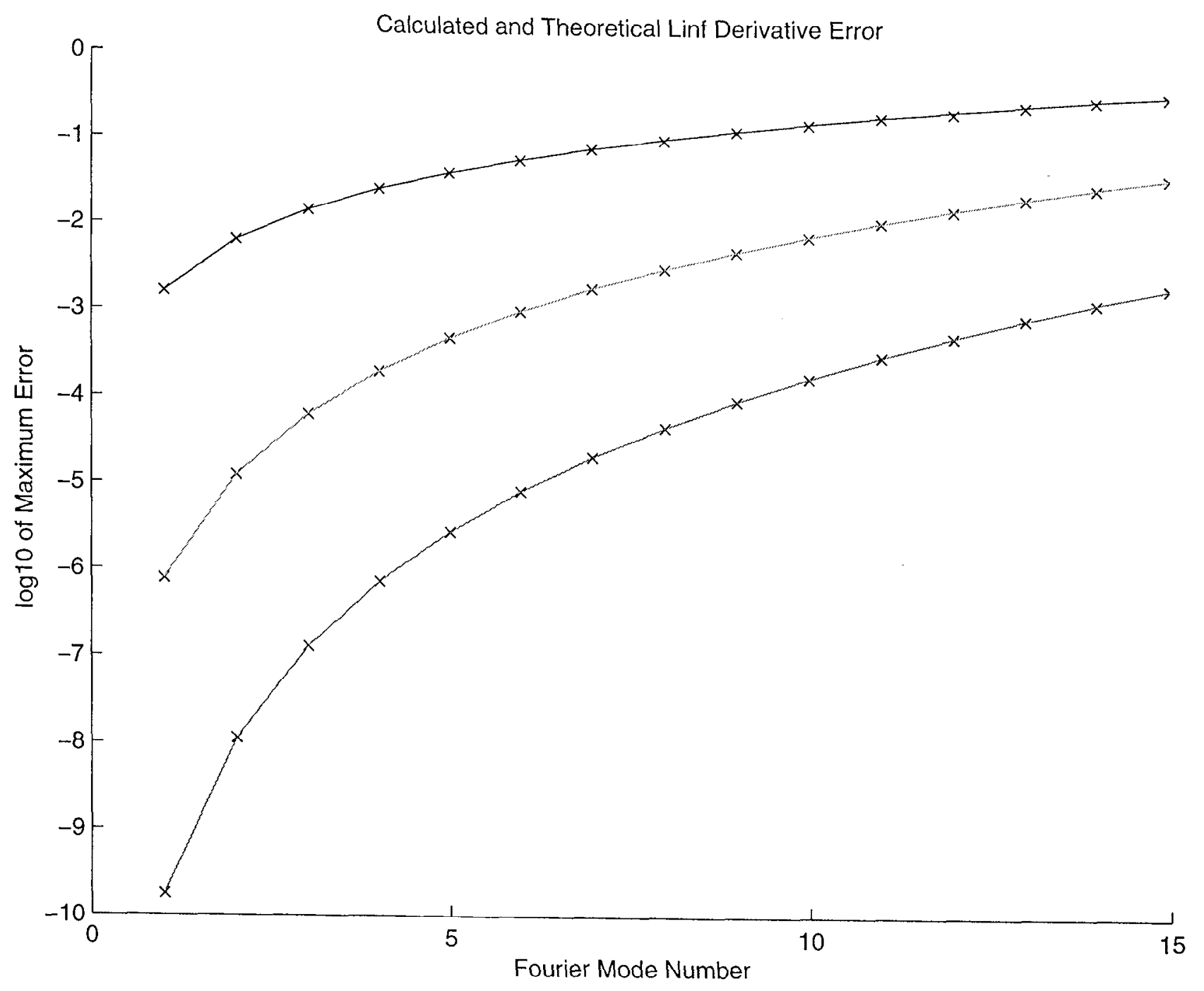

Figure 6: Error for RK2, RK4, and RK6. $c_{k}=1 / k$ Again, the spectrum is that of a shock. 


\section{Conclusion}

In this manuscript we have derived expressions which give us the maximum error that a given differentiation operator will commit for various spectra. We have confined our discussion to this operator since it is clearly the most important operator in the numerical solution of partial differential equations. It is critical to obtain this estimate of error in order to obtain an estimate of the reliability of Direct Numerical Simulation of Turbulent or other flows. 


\section{Bibliography}

\section{References}

[1] C. Canuto, M.Y. Hussaini, A. Quarteroni, T.A. Zang, (1988) "Spectral Methods in Fluid Dynamics", Springer-Verlag.

[2] L. Jameson, Numerical Errors in DNS: Total Run-Time Error. LLNL Technical Report No. XXXXX

[3] Private communication. 